\title{
The optimal duration of progesterone supplementation in pregnant women after IVF/ICSI: a meta-analysis
}

\author{
Xi-Ru Liu' ${ }^{1}$, Hua-Qiao Mu ${ }^{2}$, Qi Shi ${ }^{1}$ Xiao-Qiu Xiao ${ }^{3}$ and Hong-Bo Qi ${ }^{1 *}$
}

\begin{abstract}
Background: Progesterone supplementation after in vitro fertilisation/intracytoplasmic sperm injection (IVF/ICSI) can improve the rates of clinical pregnancy and live birth, but the optimal duration of treatment remains controversial. The objective of this meta-analysis was to investigate the effects of early progesterone cessation on pregnancy outcomes in women undergoing IVF/ICSI.

Methods: We searched MEDLINE, EMBASE, the Cochrane Central Register of Controlled Trials (CENTRAL), the Chinese biomedicine (CBM) literature database, and the Wanfang database. The final search was performed in July 2012. All available randomised trials that compared the effects of early progesterone cessation with progesterone continuation during early pregnancy after IVF/ICSI were included. The main outcome measures were live birth rate, miscarriage rate and ongoing pregnancy rate. Fixed or random-effects models were chosen to calculate the risk ratio (RR).

Results: Six eligible studies with a total of 1,201 randomised participants were included in the final analysis. No statistically significant differences were detected between patients who underwent early progesterone cessation and those who received progesterone continuation for luteal phase support in terms of live birth rate (RR: 0.95, 95\% Cl: 0.86-1.05), miscarriage rate (RR: 1.01, 95\% Cl: 0.74-1.38) or ongoing pregnancy rate (RR: $0.97,95 \%$ Cl: 0.90-1.05). These results did not change after a sensitivity analysis.

Conclusions: The currently available evidence suggests that progesterone supplementation beyond the first positive hCG test after IVF/ICSI might generally be unnecessary, although large-scale randomised controlled trials are needed to strengthen this conclusion.
\end{abstract}

Keywords: Progesterone, Luteal phase support, IVF/ICSI, Pregnancy outcome, Meta-analysis

\section{Background}

Approximately one million couples receive in vitro fertilisation (IVF) treatment every year worldwide [1]. Luteal phase support (LPS) has routinely been applied as part of this treatment. The use of agonistic or antagonistic gonadotropin-releasing hormone $(\mathrm{GnRH})$ protocols in stimulated IVF/intracytoplasmic sperm injection (ICSI) cycles cause disruptions to the luteal phase, leading to inadequate development of the endometrium and asynchrony between endometrial receptiveness and embryo

\footnotetext{
*Correspondence: qi_hongbo@yahoo.com.cn

'Department of Obstetrics and Gynaecology, The First Affiliated Hospital of Chongqing Medical University, No.1 Youyi Road, Yuzhong District, Chongqing 400016, China

Full list of author information is available at the end of the article
}

transfer. The most plausible cause of this condition is the development of multiple follicles upon ovarian stimulation, which results in superphysiological steroid concentrations and consequent inhibition of luteinising hormone (LH) secretion by the pituitary via negative feedback at the level of the hypothalamic-pituitary axis [2]. Despite the rapid recovery of the pituitary in $\mathrm{GnRH}$-antagonist protocols, luteolysisis also prematurely induced after $\mathrm{GnRH}$-antagonist co-treatment, resulting in a significant reduction in luteal phase length and a compromised reproductive outcome. For this reason, LPS remains mandatory in GnRH antagonist protocols used for IVF [3-5]. A large number of studies have shown that LPS improves the clinical pregnancy rate

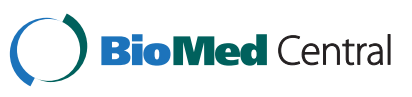


and thus the live birth rate, but the ideal LPS method remains unclear [6]. Although luteal human chorionic gonadotropin (hCG) supplementation has proven to be an effective way to overcome luteal phase defects, this treatment is frequently associated with an increased risk of ovarian hyperstimulation syndrome (OHSS) [7], so the current most widely used form of LPS is progesterone (P).

The use of $\mathrm{P}$ supplementation after oocyte retrieval (OR) is almost universal, but the optimal duration of $\mathrm{P}$ administration remains controversial. A recent large survey of 84 IVF centres in 35 countries, encompassing 51,155 cycles, found that $\mathrm{P}$ was continued until 10-12weeks of gestation in $67 \%$ of the cycles, whereas it was discontinued in $22 \%$ and $12 \%$ when foetal heart pulsations were recognised or when the $\beta$-hCG test was positive, respectively [8]. In the existing literature, $\mathrm{P}$ supplementation is variously terminated on or near the day of a positive $\beta$-hCG test [9-12] or extended to the day of the first ultrasound (5-7 weeks) [13], to the $8^{\text {th }}$ week [14-16], or as late as the $12^{\text {th }}$ week of pregnancy [17-21]. Until recently, the available data have been insufficient to determine the optimal duration of therapy, and prolonged $\mathrm{P}$ protocols have been the rule, with most clinicians following the dictum, "better safe than sorry" [8]. A growing body of evidence, however, has challenged this concept and adds to the increasing concern that $\mathrm{P}$ supplementation of early pregnancy after IVF/ICSI might be unnecessary $[10-14,22,23]$.

Four formulations of $\mathrm{P}$ are currently used for assisted reproduction, including vaginal, intramuscular (i.m.), oral and rectal preparations. Vaginal $\mathrm{P}$ was used for LPS as a single agent in $64 \%$ of cycles and in another $16 \%$ of cycles in combination with either i.m. (15\%) or oral P (1\%). As single agents, i.m. P was used in 13\% of cycles, oral P in another 2\% and hCG in 5\% [8]. Vaginal $\mathrm{P}$ can result in similar pregnancy rates as i.m. $\mathrm{P}$ and is more comfortable and tolerable to patients [24,25], but it is more expensive. Conversely, i.m. P is often associated with a number of side effects, including painful injections, severe inflammatory reactions, and sterile abscesses [26]. Prolonged and repeated i.m. injections of P in oil may also lead to delayed forms of hypersensitivity reactions, with leukocytosis, marked eosinophilia and compromised pulmonary activity $[27,28]$. Orally administered P has a first-pass effect in which a high concentration is sent to the portal circulation, which, in turn, results in the production of many liver metabolites of $\mathrm{P}$, some of which may be teratogenic. Despite the available literature on the teratogenic effects of prenatal oral P use $[29,30]$, this agent is still used routinely by many practitioners. Therefore, taking into consideration the burden of LPS treatment, the adverse reactions to $\mathrm{P}$ and updated results suggesting that $\mathrm{P}$ supplementation during early pregnancy after IVF/ICSI might be unnecessary, we questioned whether the practice of early pregnancy
P supplementation in IVF/ICSI patients should be discontinued.

The aim of this study was to perform a meta-analysis of all available randomised controlled trials (RCT) comparing early $\mathrm{P}$ cessation with $\mathrm{P}$ continuation after assisted conception in IVF/ICSI cycles to investigate potential differences in live birth, miscarriage and ongoing pregnancy rates. This review was performed in accordance with the preferred reporting items for systematic reviews and meta-analyses (PRISMA) statement principles [31].

\section{Methods}

\section{Types of studies}

The inclusion criteria for eligible studies were defined a priori during the design phase of this systematic review. Randomised controlled trials investigating the duration of $\mathrm{P}$ supplementation for luteal phase support in IVF/ ICSI cycles were included. Trials using donor oocyte cycles or frozen transfers were excluded. No limitations were placed on language, date, or publication status.

\section{Types of participants}

Women undergoing IVF/ICSI who were evaluated for the effects of $\mathrm{P}$ supplementation duration on pregnancy outcomes were included.

\section{Types of interventions}

The interventions evaluated were early $\mathrm{P}$ cessation versus $\mathrm{P}$ continuation during the first trimester in pregnant women after IVF/ICSI.

\section{Types of outcome measures}

The primary outcome chosen for the meta-analysis was live birth rate (LBR, i.e.,a baby born alive after 24 weeks gestation). Secondary outcomes included ongoing pregnancy rate (OPR, pregnancy beyond 12 weeks of gestation, as confirmed by foetal heart activity on an ultrasound), and miscarriage rate (MR, the failure to achieve live birth after a positive $\beta$-hCG test).

\section{Literature search and data collection}

We performed an exhaustive electronic search in the following databases: MEDLINE (1946 to July 2012), EMBASE (1974 to July 2012), the Cochrane Central Register of Controlled Trials (CENTRAL), the Chinese biomedicine (CBM) literature database (1978 to July 2012), and the Wanfang database (1998 to July 2012). The search combined terms and descriptors related to IVF, ICSI, luteal phase support, and progesterone. To fit with the syntax used in each consulted database, the search strategy was modified with a series of terms suggestive of RCTs as set out by the Cochrane Handbook for Systematic Review of Intervention [32](Additional file 1). No limit was placed on language. We also carefully 
browsed the references of relevant publications and added the related publications to the search. When questions related to the design or outcomes of the trials arose, we contacted the corresponding authors to confirm the information we extracted from their trials or to clarify any ambiguities.

\section{Assessment of the risk of bias in the included studies}

The risk of bias in the included studies was assessed independently by two reviewers (Xi-Ru Liu and Hua-Qiao $\mathrm{Mu}$ ) according to the guidelines recommended in the Cochrane Handbook for Systematic Review of Intervention [32]. For each study, we assessed the risk of bias related to sequence generation, allocation, blinding of participants and personnel, blinding of outcome assessment, incomplete outcome data, selective reporting and other sources of bias. A judgment of 'Yes' meant a low risk of bias, a judgment of 'No' meant a high risk of bias, and 'Unclear' indicated an unclear risk of bias. Disagreements were discussed and resolved by consensus with a third reviewer (Qi Shi).

\section{Data extraction and synthesis}

Data extraction was performed independently by two reviewers (Xi-Ru Liu and Hua-Qiao Mu). Discrepancies were resolved by discussion with a third reviewer (Qi Shi). We extracted the following information from each eligible study: first author, year of publication, country of origin, sample size, and a number of patient characteristics, including the IVF protocol used, the exact dose of P, the route of administration, the timing of initiation and duration of luteal phase support with P, and IVF/ICSI outcomes.

Raw data were extracted from the eligible studies for each defined outcome and pooled using Review Manager 5.1 software. Dichotomous results from each study were expressed as relative risk (RR) with 95\% confidence intervals (CI). These results were combined for the meta-analysis using a fixed-effects model in the absence of statistically significant heterogeneity or a randomeffects model in the presence of statistically significant heterogeneity. The inter-study heterogeneity was evaluated using the $x^{2}$ (Cochran's Q) statistic and the $I^{2}$ value. Sensitivity analyses were performed for those studies that answered the research question of interest but used a quasi-randomised approach for patient allocation. Subgroup analyses were planned a priori based on: (1) the timing of randomisation, (2) the timing of initiation of $\mathrm{P}$, (3) the GnRH analogue used for LH surge inhibition, and (4) the type and dose of P administration.

\section{Results}

Literature search results

A total of 1,185 trials were retrieved in the initial electronic search, 351 of which were duplicate records that were subsequently removed. An additional 821 were excluded upon title/abstract screening. The 13 remaining trials were selected for further full-text analysis. Seven of these trials were excluded. Two were retrospective cohort studies $[10,22]$, and one failed to implement randomisation [33]; one was the same study reported as an abstract at an earlier meeting [34]; one trial did not explicitly describe the sequence generation or allocation concealment [35]; and two trials did not meet other inclusion criteria $[18,36]$. The remaining six RCTs, totalling 1,201 participants, were included in this meta-analysis. Detailed search procedures are summarised in the flow diagram (Figure 1).

\section{The methodological quality of included studies}

Four of the six trials provided an adequate randomisation model $[11,13,14,23]$, and four an adequate mode for allocation concealment [12-14,23], all of which made use of sealed and opaque envelopes. One trial [17] used odd and even patient birth years for allocation and was classified as a quasi-randomised trial. Only one of the studies was a dual centre study [11]; the other five were unicentric. None of the studies blinded their personnel, participants or outcome assessors or at least did not mention blinding. Owing to the small number of included studies, it was impossible to conduct a meaningful assessment of publication bias using a funnel plot. See the risk of bias graph (Figure 2) and risk of bias summary (Figure 3) for an overview.

\section{Characteristics of included studies}

The six selected studies [11-14,17,23] were performed in Spain, Belgium, Minnesota (USA), Egypt, Denmark, and Germany, respectively, and involved 1,201 participants who were originally studied between 1989 and 2010. In two studies, patients with a clinical pregnancy (at 5-7 weeks of gestation) were included $[13,14]$, and in three studies, patients with a positive $\beta$-hCG test (on the 11$16^{\text {th }}$ day post-embryo transfer (ET)) were included $[11,17,23]$. In the final study, patients were enrolled at the beginning of an IVF cycle [12]. The type and dose of $P$ supplementation, the timing of administration and the duration of $\mathrm{P}$ supplementation varied among the studies. In addition, controlled ovarian hyperstimulation $(\mathrm{COH})$ protocols and the basal clinical characteristics of the patients differed between studies. These data are presented in Table 1.

\section{Live birth rate}

Two eligible studies presented data on live birth rates $[11,12]$. In the study reported by Goudge et al. [12], the number of patients recruited at the beginning of an IVF cycle was converted to the number of patients with a positive $\beta$-hCG test according to the reported biochemical pregnancy rate. There were 143 events in the early $\mathrm{P}$ 


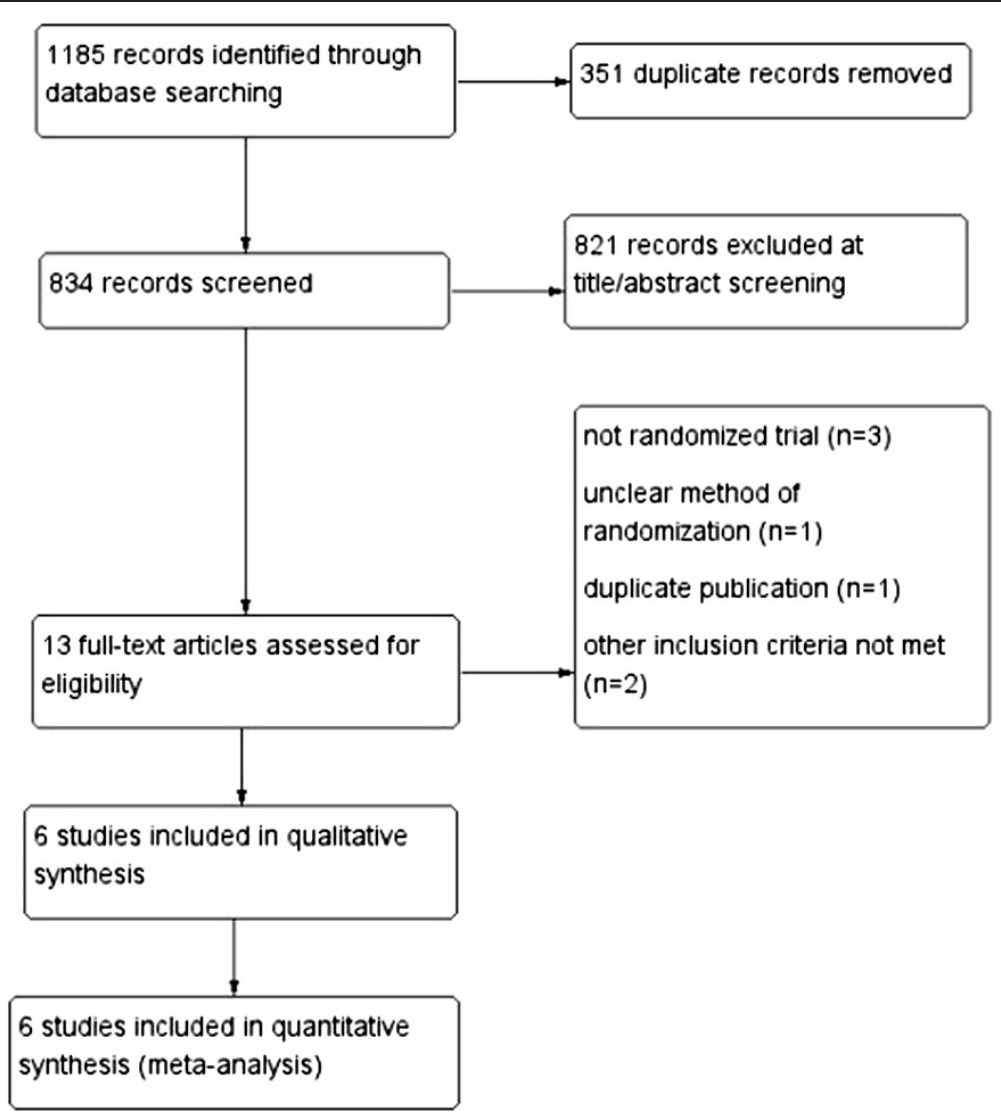

Figure 1 Flow diagram of the systematic review and meta-analysis.

cessation group (in which $\mathrm{P}$ was stopped on the $11^{\text {th }}$ or $14^{\text {th }}$ day post-ET) and 150 in the $\mathrm{P}$ continuation group (in which $\mathrm{P}$ was continued until the $6^{\text {th }}$ or $7^{\text {th }}$ week of gestation). There were a total of 293 patients who gave birth to live babies out of 369 participants. The probability of live birth did not differ between the early P cessation group $(77.3 \%, 143 / 185)$ and the $\mathrm{P}$ continuation group (81.5\%, 150/184) (P = 0.33; RR: 0.95, 95\% CI: 0.86-1.05). There was no statistical heterogeneity in this comparison $\left(\mathrm{X}^{2}=0.05, \mathrm{df}=1, \mathrm{P}=0.82 ; \mathrm{I}^{2}=0 \%\right)$ (Figure 4).

\section{Miscarriage rate}

MR data were available from six studies, with 136 events out of 1166 participants; after data conversion, this figure corresponded to $69 / 585$ in the early $\mathrm{P}$ cessation group and $67 / 581$ in the P continuation group [11-14,17,23]. No statistical heterogeneity was observed between the studies

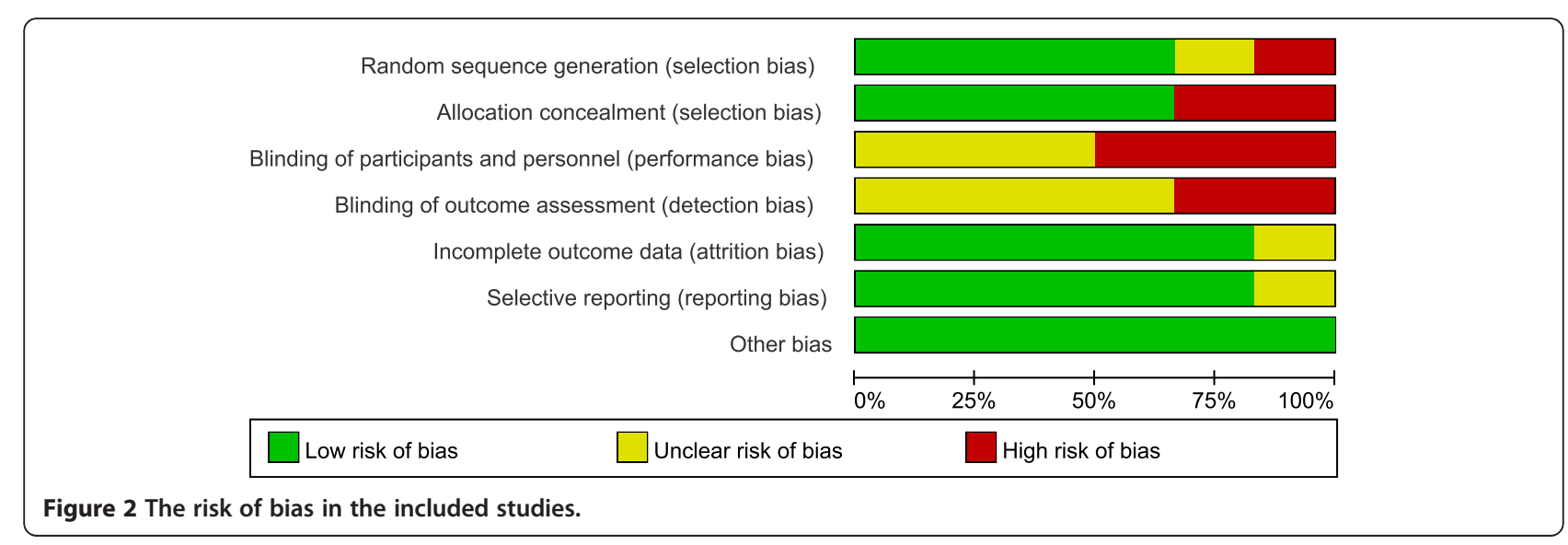




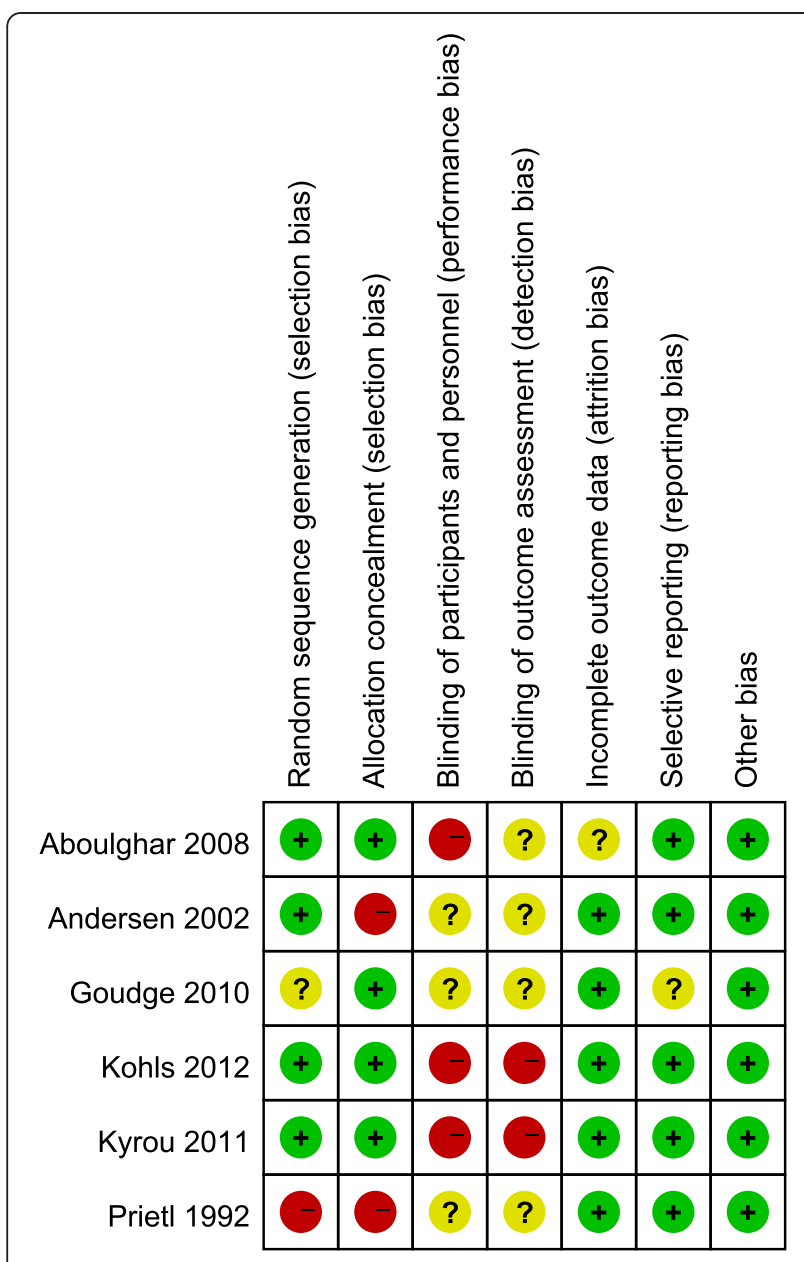

Figure 3 Summary of the risk of bias in the included studies.

$\left(x^{2}=2.96, \mathrm{df}=5, \mathrm{P}=0.71 ; \mathrm{I}^{2}=0 \%\right)$. There were no significant differences in the number of miscarriages between patients who received early $\mathrm{P}$ cessation and those who received $\mathrm{P}$ continuation ( $\mathrm{P}=0.96$; $\mathrm{RR}$ : $1.01,95 \% \mathrm{CI}$ : 0.74-1.38) (Figure 5).

\section{Ongoing pregnancy rate}

OPR data were available from six studies, with 1017 events among 1166 participants (503/585 in the early $\mathrm{P}$ cessation group and 514/581 in the P continuation group) [11-14,17,23]. A meta-analysis of all six trials yielded an RR of 0.97 ( $P=0.49$; 95\% CI: 0.90-1.05), indicating that there was no statistically significant difference between the early $\mathrm{P}$ cessation and $\mathrm{P}$ continuation groups (Figure 6).

Because the OPR was heterogeneous $\left(X^{2}=18.75, \mathrm{df}=5\right.$, $\left.\mathrm{P}=0.002 ; \mathrm{I}^{2}=73 \%\right)$ among the included studies, a random-effects model was used. No differences were observed between the results obtained using a fixedeffects model ( $\mathrm{P}=0.28$; RR: 0.98; 95\% CI: 0.94-1.02) and those obtained from the random-effects model $(\mathrm{P}=0.49$; RR: 0.97; 95\% CI: 0.90-1.05), although the weight of each study was altered. Next, according to the timing of randomisation, we performed a subgroup analysis and separately pooled four studies $[11,12,17,23]$ in which $P$ was withdrawn on the day of a positive $\beta$-hCG test and two studies $[13,14]$ in which $P$ was withdrawn on the day that clinical pregnancy was confirmed $\left(5^{\text {th }}-7^{\text {th }}\right.$ weeks of gestation). This stratified analysis revealed no significant differences between the groups in which $\mathrm{P}$ was stopped on the day of a positive $\beta$-hCG test $(\mathrm{P}=0.29$; RR: 0.91 ; 95\% CI: $0.76-1.09)$ or on the day that clinical pregnancy was verified ( $\mathrm{P}=0.55$; RR: $1.01 ; 95 \% \mathrm{CI}$ : 0.97-1.06). Heterogeneity was detected in the subgroup of studies that randomised patients on the day of a positive $\beta$-hCG test $\left(\chi^{2}=15.15, \mathrm{df}=3, \mathrm{P}=0.002 ; \mathrm{I}^{2}=80 \%\right)$. The study reported by Prietl [17] might be the source of heterogeneity, as it used a different luteal phase support protocol (17 $\alpha$-hydroxyprogesterone caproate $(100 \mathrm{mg})$ and oestradiol valerate (10mg) twice a week) and exhibited a high risk of bias based on sequence generation and patient allocation methods. In a sensitivity analysis, we recalculated the combined results while excluding this study. However, the results before ( $\mathrm{P}=0.49$; RR: 0.97; 95\% CI: 0.90-1.05)

Table 1 Characteristics of the studies included in this review

\begin{tabular}{|c|c|c|c|c|c|c|c|c|c|c|}
\hline $\begin{array}{l}\text { Study Author, } \\
\text { year }\end{array}$ & $\begin{array}{l}\text { Timing of } \\
\text { randomisation }\end{array}$ & ART & $\mathrm{COH}$ protocols & Total & $\begin{array}{l}\text { Initiation } \\
\text { of } P\end{array}$ & $\begin{array}{l}\text { Dose } \& \text { route of } \\
\text { administration }\end{array}$ & No. & $\begin{array}{l}\text { Early P } \\
\text { cessation } \\
\text { group }\end{array}$ & No. & $\begin{array}{l}\text { Continuation } \\
\text { group }\end{array}$ \\
\hline Kohls, 2012 & Clinical pregnancy & IVF/ICSI & GnRH-anta & 220 & OR & vaginal P 200mg bid & 110 & week 5 & 110 & week 8 \\
\hline Kyrou, 2011 & Positive hCG test & IVF/ICSI & GnRH-anta & 200 & ET & vaginal P 200mg tid & 100 & $\begin{array}{l}\text { the } 16^{\text {th }} \text { day } \\
\text { post-ET }\end{array}$ & 100 & week 7 \\
\hline Goudge, 2010 & $\mathrm{COH}$ & IVF & $\begin{array}{l}\text { GnRH-a/ } \\
\text { GnRH-anta }\end{array}$ & 101 & $\mathrm{ET} / \mathrm{OR}$ & IM P50mg qd & 53 & $\begin{array}{l}\text { the } 11^{\text {th }} \text { day } \\
\text { post-ET }\end{array}$ & 48 & week 6 \\
\hline Aboulghar, 2008 & Clinical pregnancy & $|C S|$ & GnRH-a & 257 & Unstated & IM or vaginal P & 125 & week 6-7 & 132 & week 9-10 \\
\hline Andersen, 2002 & Positive hCG test & IVF/ICSI & $\mathrm{GnRH}-\mathrm{a}$ & 303 & ET & vaginal P 200mg tid & 150 & $\begin{array}{l}\text { the } 14^{\text {th }} \text { day } \\
\text { post-ET }\end{array}$ & 153 & week 7 \\
\hline Prietl, 1992 & Positive hCG test & IVF & $\begin{array}{l}\text { CC/hMG/ } \\
\text { GnRH-a }\end{array}$ & 120 & Unstated & PC500mg/EV10mg tiw & 65 & $\begin{array}{l}\text { the } 12^{\text {th }} \text { day } \\
\text { post-ET }\end{array}$ & 55 & week 12 \\
\hline
\end{tabular}




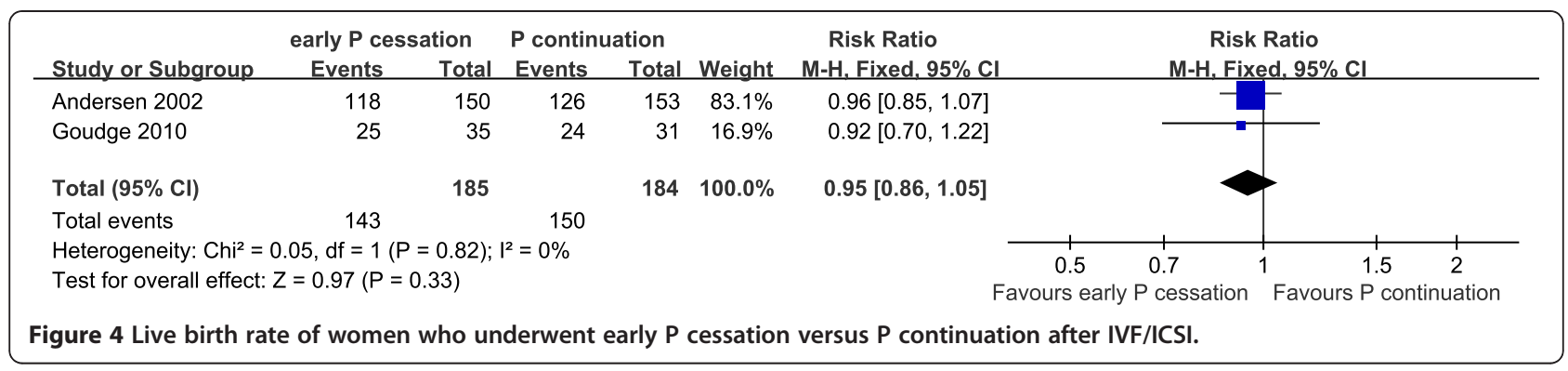

and after the sensitivity analysis $(\mathrm{P}=0.74$; RR: $1.01 ; 95 \% \mathrm{CI}$ : 0.96-1.06) were not significantly different.

\section{Discussion}

Although there is no firm evidence to support the continuation of LPS until the $10^{\text {th }}$ to $12^{\text {th }}$ week of gestation, this practice is used in the majority of IVF cycles worldwide [8]. This review compared the effects of early cessation with continuation of $\mathrm{P}$ supplementation for luteal phase support in pregnant women after IVF/ICSI, focusing on the live birth, ongoing pregnancy and miscarriage rates. The pooled results showed no significant differences in LBR between groups in which $P$ supplementation was stopped on the day of a positive $\beta$-hCG test or for whom P supplementation was continued up to the $6^{\text {th }}$ to $7^{\text {th }}$ week of gestation. Similarly, the miscarriage and ongoing pregnancy rates were not affected by the duration of $\mathrm{P}$ administration. Because there was statistical heterogeneity in the studies analysed for OPR, we performed a subgroup analysis and a sensitivity analysis. The results of the subgroup analysis were in accordance with the above results. The findings were also stable after the sensitivity analysis, which excluded one study [17] in which odd or even patient birth years were used for patient allocation. Based on this analysis, we find no convincing evidence to support the routine use of $\mathrm{P}$ supplementation during early pregnancy in women undergoing IVF/ICSI. It is possible that the establishment of a pregnancy and rescue of the corpus luteum via trophoblastic hCG may make up for the possible luteal phase defect caused by the stimulated IVF cycles.

Most of the studies included in this review described their methods of sequence generation and allocation concealing. However, none of the studies mentioned blinding. Keeping trial participants, personnel, or assessors blinded to the assigned intervention might reduce the influence of subjective psychological factors on pregnancy outcomes, an important aspect of RCTs. However, owing to the nature of current LPS studies, absolute double blinding is often not practical, as it is not possible to blind the participants. None of the studies explicitly mentioned blinding of personnel or outcome assessors. Nevertheless, it is unlikely that pregnancy outcomes such as live birth, miscarriage or ongoing pregnancy can be affected by detection bias. In future studies, proper blinding protocols using a double-dummy design should be implemented, and a placebo control group should be established.

Due to the small number of studies that met the inclusion criteria and the different clinical characteristics of the participants, it was impossible to conduct meaningful subgroup analyses based on the initiation of P supplementation, the $\mathrm{GnRH}$ analogue used for luteinising hormone surge inhibition, or the type or dose of $\mathrm{P}$ administration. These analyses might become practical upon the accumulation of further studies. We were only able to analyse studies according to the different timing of randomisation, a potential source of clinical heterogeneity; here, we pooled the data from studies with similar enrolment

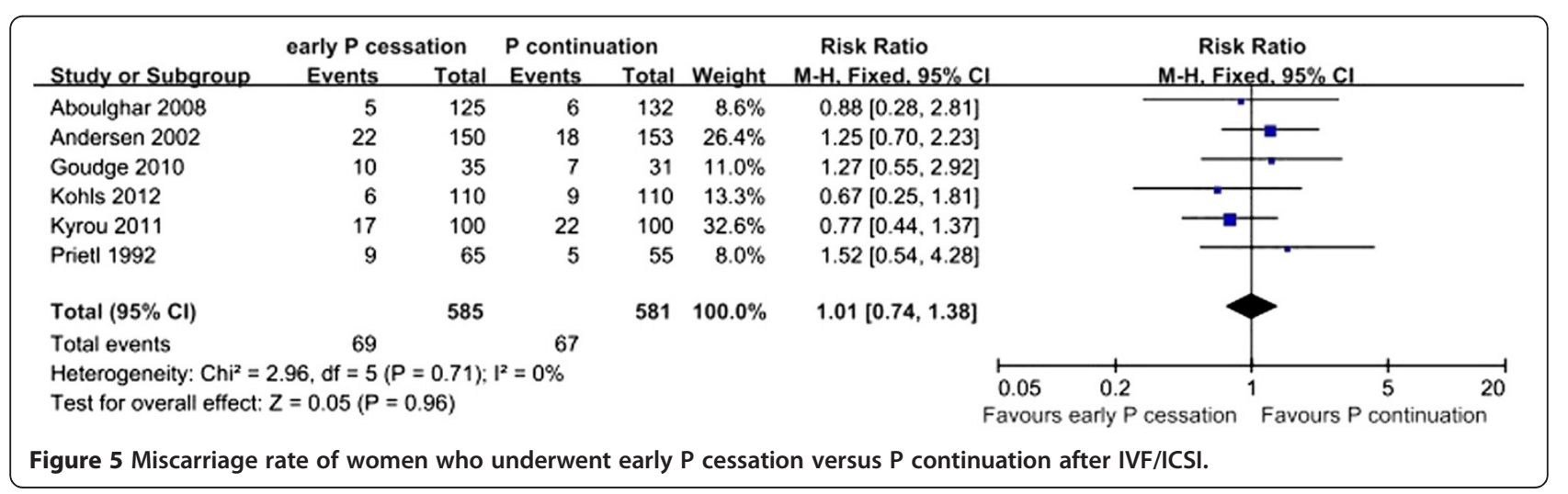


designs. However, it should be noted that the aforementioned parameters do vary among the included studies, and we do not know whether these clinical variables might be associated with the effects of $P$ supplementation on pregnancy outcomes. For example, $\mathrm{P}$ supplementation was initiated at different time-points in these studies. These were no significant differences between initiation on the day of OR or on the day of ET with regard to clinical pregnancy, ongoing pregnancy, or live birth rates, according to recent reports $[36,37]$. One study suggested that delaying the LPS until six days after OR can decrease the pregnancy rate [38]. Because $P$ administration was initiated on the day of OR or ET in most of the eligible studies in our analysis, it is unlikely that these discrepancies affected our results. In a word, our results require confirmation in further studies in which the baseline of different groups is comparable to the greatest possible extent.

Two important limitations of our meta-analysis should be noted: (1) only six studies were included in this review, and the number of patients analysed is far below the sample size required to exclude a clinically important difference. A non-inferiority trial showing a difference of $-4 \%$ or larger from a live birth rate of $80 \%$ would require a sample size of 3,140 women with a positive pregnancy test after IVF [39]. (2) The external validity of the study may be limited because existing studies excluded those patients with early bleeding, advanced age or polycystic ovary syndrome as well as those patients with an inadequate hCG rise or endometriosis, whose luteal phase may behave differently. Future trials should recruit such patients to better stratify the outcomes for these patient groups.

\section{Conclusions}

In conclusion, based on the currently available evidence, progesterone supplementation might be unnecessary beyond the first positive $\beta$-hCG test after IVF/ICSI.
However, considering the large number of IVF cycles performed globally and the side effects and costs of progesterone treatment, additional well-designed RCTs are urgently needed to investigate the optimal duration of progesterone administration during early pregnancy in women undergoing IVF/ICSI.

\section{Additional file}

Additional file 1: Search strategy in MEDLINE, EMBASE, and CENTRAL databases.

\section{Abbreviations}

$\mathrm{COH}$ : Controlled ovarian hyperstimulation; ET: Embryo transfer; $\mathrm{GnRH}$ : Gonadotropin-releasing hormone; hCG: human chorionic gonadotropin; IVF: In vitro fertilisation; ICSI: Intracytoplasmic sperm injection; i.m: intramuscular; LBR: Live birth rate; LH: Luteinising hormone; LPS: Luteal phase support; MR: Miscarriage rate; OR: Oocyte retrieval; OPR: Ongoing pregnancy rate; P: Progesterone; RCT: Randomised controlled trials.

\section{Competing interests}

The authors declare that they have no competing interests.

\section{Authors' contributions}

Hong-Bo Qi was responsible for designing and coordinating the study. All authors were responsible for data collection, data analysis, and data interpretation. Xi-Ru Liu, Hua-Qiao Mu, and Qi Shi were responsible for the statistical analysis and for writing the manuscript. Xiao-Qiu Xiao was responsible for reviewing the manuscript. All authors read and approved the final manuscript.

\section{Acknowledgements}

The authors wish to thank the ELSEVIER WebShop experts for revising the English text.

\section{Author details}

${ }^{1}$ Department of Obstetrics and Gynaecology, The First Affiliated Hospital of Chongqing Medical University, No.1 Youyi Road, Yuzhong District, Chongqing 400016, China. ${ }^{2}$ Chongqing Health Centre for Women and Children, No.64 Jintang Street, Yuzhong District, Chongqing 400013, China. ${ }^{3}$ Laboratory of Lipid \& Glucose Research, The First Affiliated Hospital of Chongqing Medical University, No.1 Youyi Road, Yuzhong District, Chongqing 400016, China. 
Received: 14 September 2012 Accepted: 8 December 2012

Published: 13 December 2012

\section{References}

1. Zegers-Hochschild F, Adamson GD, de Mouzon J, Ishihara O, Mansour R, Nygren K, Sullivan E, Vanderpoel S: International Committee for Monitoring Assisted Reproductive Technology (ICMART) and the World Health Organization (WHO) revised glossary of ART terminology. Fertil Steril 2009, 92:1520-1524.

2. Humaidan P, Papanikolaou EG, Kyrou D, Alsbjerg B, Polyzos NP, Devroey P, Fatemi HM: The luteal phase after $\mathrm{GnRH}$-agonist triggering of ovulation: present and future perspectives. Reprod Biomed Online 2012, 24:134-141.

3. Beckers NG, Macklon NS, Eijkemans MJ, Ludwig M, Felberbaum RE, Diedrich K, Bustion S, Loumaye E, Fauser BC: Nonsupplemented luteal phase characteristics after the administration of recombinant human chorionic gonadotropin, recombinant luteinising hormone, or gonadotropinreleasing hormone $(\mathrm{GnRH})$ agonist to induce final oocyte maturation in in vitro fertilization patients after ovarian stimulation with recombinant follicle-stimulating hormone and GnRH antagonist cotreatment. J Clin Endocrinol Metab 2003, 88:4186-4192.

4. Dal Prato L, Borini A: Use of antagonists in ovarian stimulation protocols. Reprod Biomed Online 2005, 10:330-338.

5. Tarlatzis BC, Fauser BC, Kolibianakis EM, Diedrich K, Rombauts L, Devroey P: $\mathrm{GnRH}$ antagonists in ovarian stimulation for IVF. Hum Reprod Update 2006, 12:333-340.

6. van der Linden M, Buckingham K, Farquhar C, Kremer JA, Metwally M: Luteal phase support for assisted reproduction cycles. Cochrane database of systematic reviews (Online) 2011, 10:CD009154.

7. Soliman S, Daya S, Collins J, Hughes EG: The role of luteal phase support in infertility treatment: A meta-analysis of randomized trials. Fertil Steril 1994, 61:1068-1076

8. Vaisbuch E, Leong M, Shoham Z: Progesterone support in IVF: is evidencebased medicine translated to clinical practice? A worldwide web-based survey. Reprod Biomed Online 2012, 25:139-145.

9. Mochtar $\mathrm{MH}$, Hogerzeil HV, Mol BW: Progesterone alone versus progesterone combined with HCG as luteal support in GnRHa/HMG induced IVF cycles: a randomized clinical trial. Hum Reprod 1996, 11:1602-1605.

10. Schmidt KL, Ziebe S, Popovic B, Lindhard A, Loft A, Andersen AN: Progesterone supplementation during early gestation after in vitro fertilization has no effect on the delivery rate. Fertil Steril 2001, 75:337-341.

11. Andersen AN, Popovic-Todorovic B, Schmidt KT, Loft A, Lindhard A, Hojgaard A, Ziebe S, Hald F, Hauge B, Toft B: Progesterone supplementation during early gestations after IVF or ICSI has no effect on the delivery rates: A randomized controlled trial. Hum Reprod 2002, 17:357-361.

12. Goudge CS, Nagel TC, Damario MA: Duration of progesterone-in-oil support after in vitro fertilization and embryo transfer: A randomized, controlled trial. Fertil Steril 2010, 94:946-951.

13. Aboulghar MA, Amin YM, Al-Inany HG, Aboulghar MM, Mourad LM, Serour GI, Mansour RT: Prospective randomized study comparing luteal phase support for ICSI patients up to the first ultrasound compared with an additional three weeks. Hum Reprod 2008, 23:857-862.

14. Kohls G, Ruiz F, Martinez M, Hauzman E, de la Fuente G, Pellicer A, GarciaVelasco JA: Early progesterone cessation after in vitro fertilization/ intracytoplasmic sperm injection: a randomized, controlled trial. Fertil Steril 2012, 98:858-862.

15. Polson DW, Rogers PA, Krapez JA, Leeton JF: Vaginal progesterone as luteal phase support in an IVF/GIFT programme. Eur J Obstet Gynecol Reprod Biol 1992, 46:35-38.

16. Miles RA, Paulson RJ, Lobo RA, Press MF, Dahmoush L, Sauer MV: Pharmacokinetics and endometrial tissue levels of progesterone after administration by intramuscular and vaginal routes: a comparative study. Fertil Steril 1994, 62:485-490.

17. Prietl G, Diedrich K, van der Ven HH, Luckhaus J, Krebs D: The effect of 17 alpha-hydroxyprogesterone caproate/oestradiol valerate on the development and outcome of early pregnancies following in vitro fertilization and embryo transfer: a prospective and randomized controlled trial. Hum Reprod 1992, 7:1-5.

18. Ludwig $M$, Diedrich $K$ : Evaluation of an optimal luteal phase support protocol in IVF. Acta Obstet Gynecol Scand 2001, 80:452-466.

19. Smitz J, Erard P, Camus M, Devroey P, Tournaye H, Wisanto A, Van Steirteghem AC: Pituitary gonadotrophin secretory capacity during the luteal phase in superovulation using GnRH-agonists and HMG in a desensitization or flare-up protocol. Hum Reprod 1992, 7:1225-1229.

20. Van Steirteghem AC, Smitz J, Camus M, Van Waesberghe L, Deschacht J, Khan I, Staessen C, Wisanto A, Bourgain C, Devroey P: The luteal phase after invitro fertilization and related procedures. Hum Reprod 1988, 3:161-164.

21. Schoolcraft WB, Hesla JS, Gee MJ: Experience with progesterone gel for luteal support in a highly successful IVF programme. Hum Reprod 2000, 15:1284-1288.

22. Proctor A, Hurst BS, Marshburn PB, Matthews ML: Effect of progesterone supplementation in early pregnancy on the pregnancy outcome after in vitro fertilization. Fertil Steril 2006, 85:1550-1552.

23. Kyrou D, Fatemi HM, Zepiridis L, Riva A, Papanikolaou EG, Tarlatzis BC, Devroey P: Does cessation of progesterone supplementation during early pregnancy in patients treated with recFSH/GnRH antagonist affect ongoing pregnancy rates? A randomized controlled trial. Hum Reprod 2011, 26:1020-1024.

24. Polyzos NP, Messini Cl, Papanikolaou EG, Mauri D, Tzioras S, Badawy A, Messinis IE: Vaginal progesterone gel for luteal phase support in IVF/ICSI cycles: A meta-analysis. Fertil Steril 2010, 94:2083-2087.

25. Doody K, Bush M, Collins M: Progesterone supplementation for luteal support: Efficacy and patient experiences with vaginal inserts (Endometrin(registered trademark)) versus intramuscular injection. Fertil Steril 2012, 97:S18.

26. Propst AM, Hill JA, Ginsburg ES, Hurwitz S, Politch J, Yanushpolsky EH: A randomized study comparing Crinone $8 \%$ and intramuscular progesterone supplementation in in vitro fertilization-embryo transfer cycles. Fertil Steril 2001, 76:1144-1149.

27. Phy JL, Weiss WT, Weiler CR, Damario MA: Hypersensitivity to progesterone-in-oil after in vitro fertilization and embryo transfer. Fertil Steril 2003, 80:1272-1275.

28. Veysman B, Vlahos I, Oshva L: Pneumonitis and eosinophilia after in vitro fertilization treatment. Ann Emerg Med 2006, 47:472-475.

29. Carmichael SL, Shaw GM, Laurent C, Croughan MS, Olney RS, Lammer EJ: Maternal progestin intake and risk of hypospadias. Arch Pediatr Adolesc Med 2005, 159:957-962.

30. Silver Rl: Endocrine abnormalities in boys with hypospadias. Adv Exp Med Biol 2004, 545:45-72

31. Liberati A, Altman DG, Tetzlaff J, Mulrow C, Gotzsche PC, loannidis JP, Clarke M, Devereaux PJ, Kleijnen J, Moher D: The PRISMA statement for reporting systematic reviews and meta-analyses of studies that evaluate healthcare interventions: explanation and elaboration. BMJ 2009, 339:b2700.

32. Higgins JPT, Green S: Cochrane Handbook for Systematic Reviews of Interventions Version 5.1.0 [updated March 2011], The Cochrane Collaboration 2011 [www.cochrane-handbook.org].

33. Stovall DW, Van Voorhis BJ, Sparks AE, Adams LM, Syrop CH: Selective early elimination of luteal support in assisted reproduction cycles using a gonadotropin-releasing hormone agonist during ovarian stimulation. Fertil Steril 1998, 70:1056-1062.

34. Kohls G, Ruiz FJ, De La Fuente G, Toribio M, Martinez M, Pellicer A, GarciaVelasco JA: Early progesterone cessation after in vitro fertilization. Hum Reprod 2010, 25:i249.

35. ZHAO X, CHEN Z, Hong Y: Evaluation of Luteal Support after IVF-ET. Journal of Reproduction and Contraception (Chinese) 2005, 25:410-413.

36. Mochtar MH, Van Wely M, Van der Veen F: Timing luteal phase support in $\mathrm{GnRH}$ agonist down-regulated IVF/embryo transfer cycles. Hum Reprod 2006, 21:905-908.

37. Baruffi R, Mauri AL, Petersen CG, Felipe V, Franco JG Jr: Effects of vaginal progesterone administration starting on the day of oocyte retrieval on pregnancy rates. J Assist Reprod Genet 2003, 20:517-520.

38. Williams SC, Oehninger S, Gibbons WE, Van Cleave WC, Muasher SJ: Delaying the initiation of progesterone supplementation results in decreased pregnancy rates after in vitro fertilization: a randomized, prospective study. Fertil Steril 2001, 76:1140-1143.

39. Griesinger G: Editorial commentary: is it time to abandon progesterone supplementation of early pregnancy after IVF? Hum Reprod 2011, 26:1017-1019.

\section{doi:10.1186/1477-7827-10-107}

Cite this article as: Liu et al:: The optimal duration of progesterone supplementation in pregnant women after IVF/ICSI: a meta-analysis. Reproductive Biology and Endocrinology 2012 10:107. 